\title{
含吡唑环的 1,3,4-噁二唑类衍生物的合成及杀菌活性研究
}

\author{
孙娜波 $* a$ 童建颖 ${ }^{a}$ 武宏科*,b \\ ( ${ }^{a}$ 浙江树人大学生物与环境学院 杭州 310015) \\ ( ${ }^{b}$ 浙江工业大学化学工程与材料学院 杭州 310014)
}

\begin{abstract}
摘要 以乙酰丙酮、水合肼等为原料, 通过多步反应制备了一系列新型含吡唑环的 1,3,4-噁二唑类衍生物, 并考察了微 波辐射对反应的影响. 产物结构均经过 ${ }^{1} \mathrm{H}$ NMR, MS 和元素分析确证; 对所有化合物进行了活体杀菌活性测试, 结果 表明大部分化合物对黄瓜褐斑病、黄瓜核菌病、黄瓜霜霉病、黄瓜细菌性角斑病均具有较好的防效.

关键词 1,3,4-噁二唑; 吡唑; 合成; 杀菌活性
\end{abstract}

\section{Synthesis and Fungicidal Activity of 1,3,4-Oxadiazole Derivatives Containing Pyrazole Moiety}

\author{
Sun, Nabo ${ }^{*, a}$ Tong, Jianying ${ }^{a} \quad$ Wu, Hongke ${ }^{*, b}$ \\ $\left({ }^{a}\right.$ College of Biology and Environmental Engineering, Zhejiang Shuren University, Hangzhou 310015) \\ $\left({ }^{b}\right.$ College of Chemical Engineering and Materials Science, Zhejiang University of Technology, Hangzhou 310014)
}

\begin{abstract}
A series of novel 1,3,4-oxadiazole derivatives were synthesized from acetylacetone, hydrazine hydrate by multi-step reactions. Their structures were characterized by ${ }^{1} \mathrm{H}$ NMR, MS and elemental analyses. The target compounds were evaluated for their fungicidal activities against Corynespora cassiicola, Pseudomonas syringae pv. Lachrymans, Pseudoperonospora cubensis and Sclerotinia sclerotiorum in vivo, and the results indicated that some of the title compounds displayed excellent fungicidal activities.
\end{abstract}

Keywords 1,3,4-oxadiazole; pyrazole; synthesis; fungicidal activity

杂环化合物是很多天然化合物和合成医药农药中 的核心结构单元，因其具有高效低毒及其环上的取代基 可以多方位变换而逐渐成为医药农药领域的研究热 点 ${ }^{[1 \sim 5]}$. 吡唑环作为杂环化合物中的一个重要分支, 其 衍生物表现出广泛的生物活性, 如杀菌 ${ }^{[6]}$ 、杀虫 ${ }^{[7]} 、$ 除

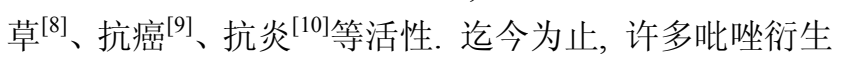
物已被国内外公司成功开发为农药和医药. 如杀虫剂吡 螨胺 (Tebufenpyrad), 氯虫酰胺 (Chlorantraniliprole) 等; 杀菌剂吡噻菌胺 (penthiopyrad), 唑菌胺酯 (Pyraclostrobin)等; 镇痛、消炎、退热药物的安替比林(Antipyrine)等. 1,3,4-留二唑类化合物因具有除草 ${ }^{[11]}$ 、杀菌 ${ }^{[12]}$ 、 杀虫 ${ }^{[13]}$ 、抗癌 ${ }^{[14]}$ 、抗炎 ${ }^{[15]}$ 等多样性的生物活性而同样倍 受农药化学家和药物学家的关注.

我们课题组在前期工作 ${ }^{[16]}$ 的基础上，根据活性亚 结构拼接、生物等排等方法, 将活性功能团吡唑官能团
和 1,3,4-噁二唑以及含 $\mathrm{SCH}_{2}$ 官能团合并在同一分子中 设计合成标题化合物, 其结构经 ${ }^{1} \mathrm{H}$ NMR, MS 和元素 分析确证. 初步生物活性测试结果表明部分化合物对测 试菌种具有优异的防效. 目标化合物的合成路线如 Scheme 1 所示.

\section{1 实验部分}

\section{1 试剂与仪器}

试剂均采用分析纯试剂. 未经处理, 直接使用.

北京泰克仪器有限公司 X-4 数字显示显微熔点仪, 温度计未校正; BRUKER AV-400 型核磁共振仪(400 $\mathrm{MHz})\left(\mathrm{CDCl}_{3}\right.$ 为溶剂, TMS 为内标); Thermo Finnigan LCQ Advantage 型质谱仪; Yanaco MT-3CHN 元素分析 仪; 美国 CEM 公司 Discovery ${ }^{\mathrm{TM}}$ 微波合成仪.

\footnotetext{
*E-mail: wuhk910@gmail.com; nabosun@gmail.com

Received August 20, 2012; revised October 7, 2012; published online October 12, 2012.

Project supported by the Program of Education Department of Zhejiang Province (No. Y200803060).

浙江省教育厅基金(No. Y200803060)资助项目.
} 


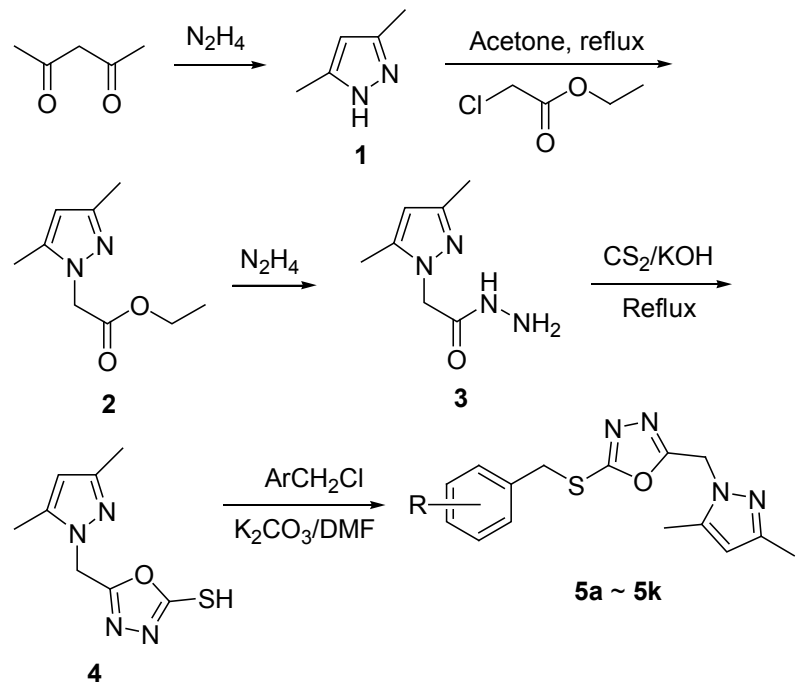

Scheme 1

\section{2 化合物的合成}

2,5-二甲基吡唑参考文献[6]方法由乙酰丙酮和水合 肼等合成.

\subsubsection{2-(3,5-二甲基- $1 H$-吡唑-1-基)乙酸乙酯(2)的合} 成

$100 \mathrm{~mL}$ 三口瓶中加入 $50 \mathrm{mmol}$ 化合物 3,5-二甲基吡 唑、 $50 \mathrm{mmol}$ 无水碳酸钾、 $30 \mathrm{~mL}$ 无水丙酩和催化量的 TBAB，搅拌下缓慢滴加氯乙酸乙酯 $(51 \mathrm{mmol})$, 回流 18 $\mathrm{h}, \mathrm{TLC}$ 检测反应(碘熏显色). 冷却, 过滤, 脱溶后得褐 色液体, 对粗产物进行硅胶柱层析 $[V$ (乙酸乙酯)： $V$ (石 油醚 $)=1: 1]$, 得到 $5.01 \mathrm{~g}$ 无色透明液体, 产率 $55 \% .{ }^{1} \mathrm{H}$ NMR (400 MHz, $\left.\mathrm{CDCl}_{3}\right) \delta: 5.85$ (s, PyH, 1H), 4.75 (s, $\mathrm{NCH}_{2}, 2 \mathrm{H}$ ), 4.21 (q, $J=7.2 \mathrm{~Hz}, \mathrm{OCH}_{2} \mathrm{CH}_{3}, 2 \mathrm{H}$ ), 2.21 (s, Py- $\mathrm{CH}_{3}, 3 \mathrm{H}$ ), 2.20 (s, Py- $\left.\mathrm{CH}_{3}, 3 \mathrm{H}\right), 1.27$ (q, $J=7.3 \mathrm{~Hz}$, $\left.\mathrm{OCH}_{2} \mathrm{CH}_{3}, 3 \mathrm{H}\right)$.

\subsubsection{2-(3,5-二甲基- $1 H$-吡唑-1-基)乙酰肼(3)的合成}

$20 \mathrm{mmol}$ 的化合物 2 和 $12 \mathrm{~mL}$ 无水乙醇加入到 50 $\mathrm{mL}$ 圆底烧瓶中, 常温摚拌至固体完全溶化, 缓慢滴入 $30 \mathrm{mmol}$ 的 $100 \%$ 水合肼, 立即出现大量针状白色固体, 继续在常温下搅拌 $2 \mathrm{~h}$, 过滤, 用少量乙醇冲洗, 得 2.91 $\mathrm{g}$ 白色针状晶体, 产率 86.4\%. m.p. 199 202 ${ }^{\circ} \mathrm{C}$.

1.2.3 5-[(3,5-二甲基- $1 H$-吡唑-1-基)甲基]-1,3,4-惡二 唑-2-硫醇(4)的合成

参考文献[17]的方法合成. 在 $100 \mathrm{~mL}$ 烧瓶中加入 $0.06 \mathrm{~mol}$ 酰肼 3, $0.09 \mathrm{~mol} \mathrm{KOH}, 50 \mathrm{~mL}$ 乙醇, $10 \mathrm{~mL}$ 水, 搅拌下加入 $0.09 \mathrm{~mol} \mathrm{CS}_{2}$, 回流 $14 \mathrm{~h}$; 完毕后, 除去大部 分溶剂, 残留物倒入 $200 \mathrm{~mL}$ 水中, 加入 $10 \%$ 盐酸至 $\mathrm{pH}$ 5 6, 析出大量固体, 过滤, 滤饼经 $90 \%$ 乙醇重结晶后 得纯品, $9.54 \mathrm{~g}$ 浅黄色固体, 产率 $75.6 \%$.
1.2.4 2-取代芐硫基-5-[(3,5-二甲基-1H-吡唑-1-基)甲 基]-1,3,4-噁二唑 $(\mathbf{5 a} \sim \mathbf{5 k})$ 的合成通法

$25 \mathrm{~mL}$ 圆底烧瓶中加入 $2 \mathrm{mmol}$ 化合物 4, $2 \mathrm{mmol}$ 取 代氯茮, $8 \mathrm{~mL} N, N^{\prime}$-二甲基甲酰胺(DMF)和 $2.4 \mathrm{mmol}$ 的 $\mathrm{K}_{2} \mathrm{CO}_{3}$, 室温搅拌, 薄层色谱(TLC)检测反应进度. 反应 完毕后，反应液倾入 $30 \mathrm{~mL}$ 冰水中，析出固体，过滤， 干燥, 乙醇重结晶的产物.

2-苠硫基-5-[(3,5-二甲基- $1 H$-吡唑-1-基)甲基]-1,3,4啞二唑(5a): 白色晶体，产率 67.1\%. m.p. 64 65 ${ }^{\circ} \mathrm{C} ;{ }^{1} \mathrm{H}$ NMR $\left(\mathrm{CDCl}_{3}, 400 \mathrm{MHz}\right) \delta: 7.29 \sim 7.38(\mathrm{~m}, 5 \mathrm{H}, \mathrm{ArH})$, 5.86 (s, 1H, PyH), 5.35 (s, 2H, Py- $\left.\mathrm{CH}_{2}\right), 4.43$ (s, 2H, $\left.\mathrm{S}_{-} \mathrm{CH}_{2}\right), 2.28$ (s, 3H, Py-CH $), 2.21$ (s, 3H, Py-CH CH $_{3}$, MS (ESI) $m / z: 301[\mathrm{M}+\mathrm{H}]^{+}$. Anal. calcd for C 59.98, H 5.37, N 18.65; found C 60.21, H 5.61, N 18.51.

2-(2,4-二氯苠硫基)-5-((3,5-二甲基-1H-吡唑-1-基) 甲基)-1,3,4-弉二唑(5b): 白色晶体，产率 77.1\%. m.p. $83 \sim 84{ }^{\circ} \mathrm{C} ;{ }^{1} \mathrm{H}$ NMR $\left(\mathrm{CDCl}_{3}, 400 \mathrm{MHz}\right) \delta: 7.48$ (d, $J=$ $7.95 \mathrm{~Hz}, 1 \mathrm{H}, \mathrm{ArH}), 7.40$ (s, 1H, ArH), 7.16 (d, $J=8.22 \mathrm{~Hz}$, 1H, ArH), 5.86 (s, 1H, PyH), 5.34 (s, 2H, Py- $\mathrm{CH}_{2}$ ), 4.48 (s, $2 \mathrm{H}, \mathrm{S}-\mathrm{CH}_{2}$ ), 2.28 (s, 3H, Py-CH $\mathrm{CH}_{3}, 2.21$ (s, 3H, Py-CH ${ }_{3}$ ); MS (ESI) $m / z: 370[\mathrm{M}+\mathrm{H}]^{+}$. Anal. calcd for $\mathrm{C} 48.79, \mathrm{H}$ 3.82, N 15.17; found C 48.98, H 4.12, N 15.09.

2-(3-氯苠硫基)-5-[(3,5-二甲基- $1 H$-吡唑-1-基)甲 基]-1,3,4-惡二唑 $(\mathbf{5 c})$ ：白色晶体，产率 83.4\%. m.p. 67 $68{ }^{\circ} \mathrm{C} ;{ }^{1} \mathrm{H}$ NMR $\left(\mathrm{CDCl}_{3}, 400 \mathrm{MHz}\right) \delta: 7.39$ (s, 1H, ArH), $7.27 \sim 7.32(\mathrm{~m}, 2 \mathrm{H}, \mathrm{ArH}), 7.10 \sim 7.23(\mathrm{~m}, 2 \mathrm{H}, \mathrm{ArH}), 5.86$ (s, 1H, PyH), 5.34 (s, 2H, Py- $\left.\mathrm{CH}_{2}\right), 4.38\left(\mathrm{~s}, 2 \mathrm{H}, \mathrm{S}-\mathrm{CH}_{2}\right)$, $2.28\left(\mathrm{~s}, 3 \mathrm{H}, \mathrm{Py}-\mathrm{CH}_{3}\right), 2.21$ (s, 3H, Py-CH 3 ); $\mathrm{MS}$ (ESI) $m / z$ : $336[\mathrm{M}+\mathrm{H}]^{+}$. Anal. calcd for $\mathrm{C} 53.81, \mathrm{H} 4.52, \mathrm{~N} 16.73$; found C 53.42, H 4.89, N 16.38 .

2-(3-氧基苄硫基)-5-[(3,5-二甲基- $1 H$-吡唑-1-基)甲 基]-1,3,4-惡二唑(5d): 白色晶体，产率 85.5\%. m.p. 71 $72{ }^{\circ} \mathrm{C} ;{ }^{1} \mathrm{H}$ NMR $\left(\mathrm{CDCl}_{3}, 300 \mathrm{MHz}\right) \delta: 7.43 \sim 7.71(\mathrm{~m}, 4 \mathrm{H}$, ArH), 5.86 (s, 1H, PyH), 5.34 (s, 2H, Py-CH $), 4.23$ (s, 2H, S- $\mathrm{CH}_{2}$ ), 2.29 (s, 3H, Py- $\left.\mathrm{CH}_{3}\right), 2.21$ (s, 3H, Py-CH $)$; MS (ESI) $m / z: 326[\mathrm{M}+\mathrm{H}]^{+}$. Anal. calcd for C 59.06, H 4.65, N 21.52; found C 58.98, H 4.78, N 20.62.

2-(3-氟苠硫基)-5-[(3,5-二甲基- $1 H$-吡唑-1-基)甲 基]-1,3,4-惡二唑(5e)：白色晶体，产率 78.9\%. m.p. 72 $73{ }^{\circ} \mathrm{C} ;{ }^{1} \mathrm{H}$ NMR $\left(\mathrm{CDCl}_{3}, 300 \mathrm{MHz}\right) \delta: 6.98 \sim 7.16(\mathrm{~m}, 4 \mathrm{H}$, ArH), 5.86 (s, 1H, PyH), 5.35 (s, 2H, Py- $\left.\mathrm{CH}_{2}\right), 4.41$ (s, 2H, $\mathrm{S}_{-} \mathrm{CH}_{2}$ ), 2.29 (s, 3H, Py- $\mathrm{CH}_{3}$ ), 2.21 (s, 3H, Py-CH ${ }_{3}$; MS (ESI) $m / z: 319[\mathrm{M}+\mathrm{H}]^{+}$. Anal. calcd for C 56.59, H 4.75, N 17.60; found C 56.81, H 5.05, N 17.49.

2-(2-氯苠硫基)-5-[(3,5-二甲基- $1 H$-吡唑-1-基)甲 
基]-1,3,4-面二唑(5f)：白色晶体，产率 88.7\%. m.p. 82 $83{ }^{\circ} \mathrm{C} ;{ }^{1} \mathrm{H}$ NMR $\left(\mathrm{CDCl}_{3}, 300 \mathrm{MHz}\right) \delta: 7.51$ (d, $J=7.25$ $\mathrm{Hz}, 1 \mathrm{H}, \mathrm{ArH}), 7.38$ (d, $J=7.76 \mathrm{~Hz}, 1 \mathrm{H}, \mathrm{ArH}), 7.18 \sim 7.24$ (m, 2H, ArH), $5.86(\mathrm{~s}, 1 \mathrm{H}, \mathrm{PyH}), 5.34$ (s, $\left.2 \mathrm{H}, \mathrm{Py}-\mathrm{CH}_{2}\right)$, 4.53 (s, 2H, S-CH $\left.)_{2}\right), 2.29$ (s, 3H, Py- $\left.\mathrm{CH}_{3}\right), 2.21$ (s, 3H, Py- $\mathrm{CH}_{3}$ ); MS (ESI) $m / z: 336[\mathrm{M}+\mathrm{H}]^{+}$. Anal. calcd for $\mathrm{C}$ 53.81, H 4.52, N 16.73; found C 53.62, H 4.83, N 16.63.

2-(2-氟芐硫基)-5-[(3,5-二甲基- $1 \mathrm{H}$-吡唑-1-基)甲 基]-1,3,4-噁二唑(5g)：白色晶体, 产率 75.9\%. m.p. 91 $92{ }^{\circ} \mathrm{C} ;{ }^{1} \mathrm{H}$ NMR $\left(\mathrm{CDCl}_{3}, 300 \mathrm{MHz}\right) \delta: 7.42 \sim 7.46(\mathrm{~m}, 1 \mathrm{H}$, ArH), $7.28 \sim 7.30(\mathrm{~m}, 1 \mathrm{H}, \mathrm{ArH}), 7.03 \sim 7.11(\mathrm{~m}, 2 \mathrm{H}, \mathrm{ArH})$, $5.86(\mathrm{~s}, 1 \mathrm{H}, \mathrm{PyH}), 5.35\left(\mathrm{~s}, 2 \mathrm{H}, \mathrm{Py}-\mathrm{CH}_{2}\right), 4.46(\mathrm{~s}, 2 \mathrm{H}$, $\left.\mathrm{S}-\mathrm{CH}_{2}\right), 2.28$ (s, 3H, Py-CH $), 2.21$ (s, 3H, Py-CH $)$; MS (ESI) $m / z: 319[\mathrm{M}+\mathrm{H}]^{+}$. Anal. calcd for C 56.59, H 4.75, N 17.60; found C 56.72, H 4.35, N 17.15.

2-(2-甲基芐硫基)-5-[(3,5-二甲基- $1 H$-吡唑-1-基)甲 基]-1,3,4-留二唑(5h): 白色晶体, 产率 83.4\%. m.p. 35 $37{ }^{\circ} \mathrm{C} ;{ }^{1} \mathrm{H}$ NMR $\left(\mathrm{CDCl}_{3}, 300 \mathrm{MHz}\right) \delta: 7.31$ (d, $J=7.44$ $\mathrm{Hz}, 1 \mathrm{H}, \operatorname{ArH}), 7.13 \sim 7.21(\mathrm{~m}, 3 \mathrm{H}, \mathrm{ArH}), 5.86(\mathrm{~s}, 1 \mathrm{H}$, $\mathrm{PyH}), 5.36$ (s, 2H, Py-CH $\left.\mathrm{CH}_{2}\right), 4.46\left(\mathrm{~s}, 2 \mathrm{H}, \mathrm{SCH}_{2}\right), 2.40$ (s, $3 \mathrm{H}, \mathrm{Ar}-\mathrm{CH}_{3}$ ), 2.29 (s, 3H, Py- $\mathrm{CH}_{3}$ ), 2.21 (s, 3H, Py- $\mathrm{CH}_{3}$ ); MS (ESI) $m / z: 315[\mathrm{M}+\mathrm{H}]^{+}$. Anal. calcd for C 61.12, $\mathrm{H}$ 5.77, N 17.82; found C 60.84, H 5.70, N 17.75.

2-(4-叔丁基芐硫基)-5-((3,5-二甲基- $1 \mathrm{H}$-吡唑-1-基) 甲基)-1,3,4-噁二唑 $(\mathbf{5 i})$ : 白色晶体, 产率 $82.2 \%$. m.p. $73 \sim 74{ }^{\circ} \mathrm{C} ;{ }^{1} \mathrm{H}$ NMR $\left(\mathrm{CDCl}_{3}, 300 \mathrm{MHz}\right) \delta: 7.38$ (d, $J=$ $8.13 \mathrm{~Hz}, 2 \mathrm{H}, \mathrm{ArH}), 7.32$ (d, $J=5.32 \mathrm{~Hz}, 2 \mathrm{H}, \mathrm{ArH}), 5.86$ (s, 1H, PyH), 5.35 (s, 2H, Py-CH $_{2}, 4.58$ (s, 2H, S-CH CH $_{2}, 2.29$ (s, 3H, Py- $\mathrm{CH}_{3}$ ), 2.22 (s, 3H, Py-CH $\left.\mathrm{CH}_{3}\right), 1.30$ (s, 9H, $t$-Bu); MS (ESI) $m / z$ : $357[\mathrm{M}+\mathrm{H}]^{+}$. Anal. calcd for $\mathrm{C} 64.01, \mathrm{H}$ 6.79, N 15.72; found C 63.92, H 6.49, N 15.74.

2-(4-氟芐硫基)-5-[(3,5-二甲基- $1 H$-吡唑-1-基)甲 基]-1,3,4-留二唑(5j): 白色晶体, 产率 89.1\%. m.p. 49 $50{ }^{\circ} \mathrm{C} ;{ }^{1} \mathrm{H}$ NMR $\left(\mathrm{CDCl}_{3}, 300 \mathrm{MHz}\right) \delta: 7.33 \sim 7.37(\mathrm{~m}, 2 \mathrm{H}$, ArH), $6.96 \sim 7.00(\mathrm{~m}, 2 \mathrm{H}, \mathrm{ArH}), 5.86(\mathrm{~s}, 1 \mathrm{H}, \mathrm{PyH}), 5.34$ (s, 2H, Py- $\left.\mathrm{CH}_{2}\right), 4.39$ (s, 2H, S-CH $\left.\mathrm{CH}_{2}\right), 2.28(\mathrm{~s}, 3 \mathrm{H}$, Py- $\mathrm{CH}_{3}$ ), 2.21 (s, 3H, Py-CH 3 ); MS (ESI) $m / z: 319$ [M+ $\mathrm{H}]^{+}$. Anal. calcd for C 56.59, $\mathrm{H} 4.75, \mathrm{~N} 17.60$; found $\mathrm{C}$ $56.78, \mathrm{H} 4.68, \mathrm{~N} 17.52$.

2-(4-甲氧基芐硫基)-5-[(3,5-二甲基- $1 H$-吡唑-1-基) 甲基]-1,3,4-噁二唑(5k): 油状物, 产率 90.3\%. ${ }^{1} \mathrm{H}$ NMR $\left(\mathrm{CDCl}_{3}, 300 \mathrm{MHz}\right) \delta: 7.8 \sim 7.33$ (m, 2H, ArH), 6.82 6.89 $(\mathrm{m}, 2 \mathrm{H}, \mathrm{ArH}), 5.86(\mathrm{~s}, 1 \mathrm{H}, \mathrm{PyH}), 5.37$ (s, $\left.2 \mathrm{H}, \mathrm{Py}-\mathrm{CH}_{2}\right)$, $4.40\left(\mathrm{~s}, 2 \mathrm{H}, \mathrm{S}-\mathrm{CH}_{2}\right), 3.78$ (s, 3H, OMe), $2.26(\mathrm{~s}, 3 \mathrm{H}$, Py- $\mathrm{CH}_{3}$ ), 2.21 (s, 3H, Py-CH${ }_{3}$; MS (ESI) $m / z: 331[\mathrm{M}+$
$\mathrm{H}]^{+}$. Anal. calcd for C 58.16, H 5.49, N 16.96; found C 57.94, H 5.05, N 16.83 .

\section{3 生物活性测试}

\subsection{1 材料}

黄瓜品种: 新泰密刺播在装有基质(草炭: 蛭石为 $2: 1$ )的营养钵内, 营养钭规格 $10 \mathrm{~cm} \times 10 \mathrm{~cm}$, 每钵播 3 粒催芽种子, 保证每钭 2 株苗, 当苗子长至 2 叶 1 心时 待用.

接种体. 黄瓜霜霉病菌、黄瓜褐斑病菌、黄瓜细菌 性角斑病菌和黄瓜核菌病菌采自中国农业科学院蔬菜 花卉研究所保存, 具有较强的致病力.

\subsection{2 试验方法}

试验设在中国农业科学院蔬菜花卉研究所蔬菜病 理研究室试验玻璃温室内, 试验条件良好.

试验药剂. 所有测试样品均采用 $500 \mu \mathrm{g} / \mathrm{mL}$.

施药及接种方法: 黄瓜褐斑病菌、霜霉病菌采用狍 子悬浮液喷雾接种, 黄瓜细菌性角斑病菌采用菌悬液喷 雾接种, 黄瓜菌核病菌采用菌丝悬液喷雾接种. 于晴天 上午将供试药剂及对照药剂均匀喷施于黄瓜子叶上, $2 \mathrm{~h}$ 后接种病原菌, 保湿培养.

调查方法. 待对照充分发病后调查防效. 公式如下:

$$
\text { 防治效果 }(\%)=\frac{\text { 对照病情一处理病情 }}{\text { 对照病情 }} \times 100 \%
$$

\section{2 结果与讨论}

\section{1 合成及波谱}

化合物的合成以乙酰丙酮与水合肼反应开始，首先 制得 3,5-二甲基吡唑, 后者在相转移催化剂四丁基溴化 铵(TBAB)下, 与氯乙酸乙酯一起回流生成 2-(3,5-二甲 基- $1 H$-吡唑-1-基)乙酸乙酯(2). 中间体 2 与水合胇反应 形成 2-(3,5-二甲基-1 $H$-吡唑-1-基)乙酰肼(3); 中间体 1,3,4-噁二唑-2-硫醇 4 可以方便地由 2-(3,5-二甲基- $1 H$ 吡唑-1-基)乙酰肼(3)与 $\mathrm{KOH}$ 、二硫化碳在无水乙醇中回 流制备得到. 然后硫醇化合物与取代卤化物反应得到目 标化合物 5. 本文在合成目标化合物 $\mathbf{5}$ 时, 将中间体 $\mathbf{4}$ 与对叔丁基氯芐溶于 DMF 和水的混合溶液, 以碳酸钾 作为缚酸剂, 然后置于微波辐射下反应 $20 \mathrm{~min}$, 得到目 标化合物, 产率达到 $88 \%$, 与常规室温搅拌法产率(反应 时间为 $24 \mathrm{~h}$, 产率 $82 \%$ ) 相当, 但是反应时间大大缩短. 在 ${ }^{1} \mathrm{H}$ NMR 谱中, 连接吡唑环和噁二唑环之间的亚甲基 氢 $\delta$ 在 $5.34 \sim 5.37$ 之间; 与硫原子相连的亚甲基氢 $\delta$ 在 4.23 4.58 之间, $\mathrm{S}$ 与芐基相连时 $\delta$ 在较高场. 吡唑环上 两个甲基氢的 $\delta$ 值分别在 $\delta 2.21$ 和 2.27 左右, 且两个峰 都为单峰. 


\section{2 杀菌活性}

黄瓜褐斑病(Corynespora cassiicola)、黄瓜细菌性角 斑病(Pseudomonas syringae pv. Lachrymans)、黄瓜蔓枯 病 (Ascochyta citrullina Smith)、黄瓜霜霉病(Pseudoperonospora cubensis)和黄瓜菌核病(Sclerotinia sclerotiorum)的活体杀菌活性 ${ }^{[18,19]}$ 列于表 1 , 测试浓度为 500 $\mu \mathrm{g} / \mathrm{mL}$. 由表 1 可知, 大部分化合物在 $500 \mu \mathrm{g} / \mathrm{mL}$ 的剂量 下对黄瓜褐斑病(Corynespora cassiicola) 表现出较好的 杀菌活性, 与对照药异菌脲 (53.52\%), 井冈霉素 (78.20\%), 甲基托布津 $(78.75 \%$ )相当. 对于黄瓜褐斑病， 只有化合物 7c 表现出了中等的防效 (53.68\%), 其余化合 物的防效都在 $70 \%$ 以上，值得注意的是，化合物 $\mathbf{5 d} \sim \mathbf{5 h}$ 的防效达到了 $80 \%$ 以上，特别是化合物 $\mathbf{5 f}$ 和 $\mathbf{5 g}$ 的防效 更是超过 $90 \%$. 化合物 $5 \mathbf{a}, \mathbf{5 b}, \mathbf{5 d}$ 对黄瓜细菌性角斑病 的防效分别为 $65.59 \%, 68.08 \%, 75.63 \%$, 比对照药防效 异菌脲(38.49\%), 井冈霉素 $(-11.42 \%)$, 甲基托布津 (54.15\%)高. 对于黄瓜蔓枯病，大部分化合物的防效都 比较差, 只有化合物 5e (59.52\%)和 5g (56.81\%)具有中
等防效. 化合物 5e (68.43\%)和 5i (67.26\%)对于黄瓜霜霉 病的防效，与对照药异菌艮 (55.92\%), 井冈霉素 (56.31\%), 甲基托布津 $(68.71 \%)$ 相当. 令人意外的，部分 化合物对黄瓜菌核病反而表现出一定的促进生长作用, 如化合物 5d, 5f 和 $5 \mathrm{~g}$ 对其的抑制率分别为 $-9.10 \%$, $-10.26 \%$ 和 $-21.48 \%$. 进一步的结构优化与构效关系研 究尚在进行中.

\section{3 结论}

本文通过多步反应合成了一系列新颖的含吡唑环 的 1,3,4-噁二唑衍生物, 实验中发现利用微波合成法能 够大大缩短反应时间。活体杀菌活性实验表明，部分目 标化合物对黄瓜褐斑病、黄瓜细菌性角斑病、黄瓜霜霉 病和黄瓜菌核病有较好的防效，表明设计具有一定的合 理性. 尤其是化合物 $\mathbf{5 f}$ 和 $5 \mathrm{~g}$ 在 $500 \mathrm{mg} / \mathrm{L}$ 的剂量下对黄 瓜褐斑病的防效达 $90 \%$ 以上，优于对照药异菌艮、井冈 霉素和甲基托布津，可作为先导化合物进行进一步优化 研究.

表 1 目标化合物的活体杀菌活性 $(500 \mathrm{mg} / \mathrm{mL})$

Table 1 The fungicidal activity of title compounds in vivo $(500 \mathrm{mg} / \mathrm{mL})$

\begin{tabular}{|c|c|c|c|c|c|}
\hline Compd. & $\begin{array}{c}\text { Corynespora } \\
\text { cassiicola }\end{array}$ & $\begin{array}{c}\text { Pseudomonas syringae pv. } \\
\text { Lachrymans }\end{array}$ & $\begin{array}{l}\text { Ascochyta citrullinal } \\
\text { Smith }\end{array}$ & $\begin{array}{c}\text { Pseudoperonospora } \\
\text { cubensis }\end{array}$ & Sclerotinia sclerotiorum \\
\hline $5 a$ & 72.72 & 65.59 & 23.72 & 42.75 & 32.51 \\
\hline $5 \mathbf{b}$ & 77.66 & 68.08 & -2.73 & 41.78 & 36.93 \\
\hline $5 c$ & 53.68 & 38.02 & 19.13 & 35.97 & 12.98 \\
\hline 5d & 81.81 & 75.63 & 33.35 & 49.40 & -9.10 \\
\hline $5 e$ & 84.55 & 53.30 & 59.52 & 68.43 & 21.16 \\
\hline $5 f$ & 92.77 & 58.22 & 48.17 & 53.13 & -10.26 \\
\hline $5 g$ & 94.64 & 47.98 & 56.81 & 53.13 & -21.48 \\
\hline $5 \mathrm{~h}$ & 88.12 & 53.27 & 26.02 & 45.39 & 3.05 \\
\hline $5 \mathbf{i}$ & 62.11 & 53.52 & -29.72 & 67.26 & 33.34 \\
\hline $5 \mathbf{j}$ & 72.35 & 14.78 & 31.99 & 46.24 & 16.48 \\
\hline $\mathbf{5 k}$ & 64.26 & 32.62 & -11.66 & 59.56 & 0.81 \\
\hline 异菌脲 & 53.52 & 38.49 & 98.35 & 55.92 & 97.84 \\
\hline 井冈霉素 & 78.20 & -11.42 & 48.82 & 56.31 & 26.89 \\
\hline 甲基托布津 & 78.75 & 54.15 & 100.00 & 68.71 & 83.39 \\
\hline
\end{tabular}

致谢 本文杀菌活性测试由中国农业科学院蔬菜花卉研 究所李宝聚教授课题组完成, 特此致谢.

\section{References}

[1] Liu, X. H.; Tan, C. X.; Weng, J. Q. Phosphorus, Sulfur Silicon Relat. Elem. 2011, 186, 552.

[2] Tan, C. X.; Weng, J. Q.; Liu, Z. X.; Liu, X. H.; Zhao, W. G. Phosphorus, Sulfur Silicon Relat. Elem. 2012, 187, 990.

[3] Liu, X. H.; Weng, J. Q.; Tan, C. X. Asian J. Chem. 2011, 23, 4064.

[4] Liu, X. F.; Liu, X. H. Acta Crystallogr. 2011, E67, O202.

[5] Jiang, S. L.; Han, L. Chin. J. Org. Chem. 2012, 32, 930 (in Chinese).

(蒋绍亮，韩亮，有机化学, 2012, 32, 930.)
[6] Liu, X. H.; Tan, C. X.; Weng, J. Q. Phosphorus, Sulfur Silicon Relat. Elem. 2011, 186, 558.

[7] Sun, Y. F.; Li, Y. Q.; Ling, Y.; Yu, H. L.; Yang, S. X.; Yang, X. L. Chin. J. Org. Chem. 2011, 31, 1425 (in Chinese). (孙玉风, 李永强, 凌云, 宇红莲, 杨绍祥, 杨新玲, 有机化学, 2011, 31, 1425.)

[8] He, F. Q.; Liu, X. H.; Wang, B. L.; Li, Z. M. Heteroat. Chem. 2008, 19, 21.

[9] Puthiyapurayil, P.; Poojary, B.; Chikkanna, C.; Buridipad, S. K. Eur. J. Med. Chem. 2012, 53, 203.

[10] El-Moghazy, S. M.; Barsoum, F. F.; Abdel-Rahman, H. M.; Marzouk, A. A. Med. Chem. Res. 2012, 21, 1722.

[11] Liu, X. H.; Pan, L.; Weng, J. Q.; Tan, C. X.; Li, Y. H.; Wang, B. L.; Li, Z. M. Mol. Diversity 2012, 16, 251.

[12] Ji, Z. C.; Liu, F.; Zhang, Z. Y.; Li, F. B.; Jiang, L. Chin. J. Org. 
Chem. 2012, 32, 2129 (in Chinese).

(纪增臣, 刘峰, 张泽远, 李付博, 姜林, 有机化学, 2012, 32, 2129.)

[13] Li, X. W.; He, D. H. Chin. J. Struct. Chem. 2012, 32, 367.

[14] Singh, J.; Rajapandi, R.; Maity, T. K. Asian J. Chem. 2010, 22, 4099.

[15] Shirote, P. J.; Bhatia, M. S. Chin. J. Chem. 2010, $28,1429$.

[16] Tong, J. Y.; Shi, Y. X.; Liu, X. H.; Sun, N. B.; Li, B. J. Chin. J. Org. Chem. 2012, 32, 2373 (in Chinese).
(童建颖，石延霞，刘幸海，孙娜波，李宝聚，有机化学，2012, 32, 2373.)

[17] Jin, Y.; Song, B. A.; Hu, D. Y.; Li, X. Y.; Bhadury, P. S.; Wang, Z. C.; Yang, S. Chem. Cent. J. 2011, 5, 21.

[18] Liu, X. H.; Pan, L.; Ma, Y.; Weng, J. Q.; Tan, C. X.; Li, Y. H.; Shi, Y. X.; Li, B. J.; Li, Z. M.; Zhang, Y. G. Chem. Biol. Drug Des. 2011, 78, 689 .

[19] Tan, C. X.; Shi, Y. X.; Weng, J. Q.; Liu, X. H.; Li, B. J.; Zhao, W. G. Lett. Drug Des. Discov. 2011, 9, 431.

(Li, L.; Fan, Y.) 